\title{
Developing an Inspection Optimization Model Based on the Delay-Time Concept
}

\author{
Ehsan Nazemi and Kamran Shahanaghi \\ Department of Industrial Engineering, Iran University of Science \& Technology, Tehran, Iran \\ Correspondence should be addressed to Ehsan Nazemi; ehsan@ind.iust.ac.ir
}

Received 24 March 2015; Revised 2 June 2015; Accepted 15 June 2015

Academic Editor: Anis Chelbi

Copyright (C) 2015 E. Nazemi and K. Shahanaghi. This is an open access article distributed under the Creative Commons Attribution License, which permits unrestricted use, distribution, and reproduction in any medium, provided the original work is properly cited.

\begin{abstract}
Infrastructures are considered as important facilities required for every country and society to be able to work properly. Aging and deterioration of such structures during their lifetime are a major concern both for maintenance researchers in the academic world and for the practitioners. This concern is mainly because the deterioration increases the maintenance costs dramatically and lowers the reliability, availability, and safety of the structural system. Preventive maintenance and inspection activities are the most usual means for keeping the structure in a good condition. This paper utilizes the concept of delay-time for developing the optimal inspection policy for deteriorating structures. In the proposed stochastic model, discrete times of inspection activities are taken as the decision variables of an optimization problem, in a way that the obtained aperiodic (nonuniform) inspection schedule minimizes the total downtime ratio of the structure. To illustrate the model capabilities, various numerical examples are solved and results are compared with the traditional periodic (uniform) inspection policies. The results indicate the substantial reduction in system downtime due to the wisely planned inspection schedule and the appropriate utilization of delay-time concept, which is indeed a powerful framework for inspection optimization problems.
\end{abstract}

\section{Introduction}

Infrastructures are usually characterized by long design lifetime periods and high initial costs. Roads, railways, and highway bridges are examples of transport infrastructure and petroleum pipelines and platforms are examples of energy infrastructure. The design of such structures has been extensively studied by researchers and engineers throughout many years and is still a developing field of study. On the contrary, prediction of the condition of these systems during their lifecycle under various maintenance activities is another complex area which has not been studied until the recent decades [1].

This ever-growing research in the field of maintenance is normally because many nations have already finished public infrastructure and rushed into a maintenance period. Thus maintenance has become more important than production, manufacture, and construction [2]. In detail, as time passes and such structures become older, the system becomes less reliable and safe, and the related costs of maintenance increase so that managers and administrators in charge face more serious challenges. The situation is even more challenging when the varying circumstances dictate an extended structure lifetime while maintenance costs rise more rapidly, which is the case for many offshore oil and gas installations in the North Sea which are approaching the end of their designed lifetime period. But due to the technological improvements and higher oil prices it has been decided to continue the operations more than expected [3]. These issues explain the importance of maintenance modeling and optimization for such structures.

Maintenance optimization must initially include a proper modeling of the system in investigation. In the case of deteriorating structures, the aging phenomenon must be modeled in a suitable way. Next step is to consider the budget and resource constraints and other reasonable assumptions and finally to develop an optimization problem for the structure.

\section{Literature Review}

Christer [4] introduced the delay-time concept to justify the necessity of inspection activities for plant PM. The delay-time is a concept that divides the failure process into two stages: 
a normal working stage and a delay-time stage. In the normal working stage the system is healthy and working until an identifiable defect emerges. In the delay-time stage the system is still working but the defect causes a failure at the end; and the system fails. The key advantage of this concept is that if the distribution of the stages can be quantified, the relationship between the average number of failures and the inspection interval can be obtained. Therefore, one of the main usages and benefits of the delay-time models is to find the optimal inspection intervals. One of the early works in this area is the paper by Abdel-Hameed [5] which was corrected by himself several years later [6]. He studied an optimal periodic inspection policy model based on the class of increasing pure jump Markov processes. Thoft-Christensen and Sorensen [7] have included a reliability constraint in their model and obtained an aperiodic inspection schedule. Using the reliability index $\beta$, a basic definition in the structural reliability context, they have defined a decision tree and described possible maintenance actions after each inspection. Among the varieties of the delay-time models, Christer and Wang [8] considered a multicomponent system and added the feature of opportunity inspection to their model. However, the mathematical complexity of their model is very high and because the arrival rate of defects is not constant, a recursive algorithm was developed by them later in [9] to find the optimal nonconstant inspection intervals until the final replacement. Okumura et al. [10] and Okumura [11] proposed a method for determining discrete time points of inspection for a single unit deteriorating system, without using the stochastic processes. Their work is different from other works as they have included a failure physics model for describing the deterioration process. But similar to the other works they have used the renewal theory and minimized the long-run average cost per unit time. Wang and Christer [12] have addressed the problem of maintenance optimization for petroleum pipelines. They have calculated the failure probabilities using the structural reliability methods. Also, an elegant way of accounting for the inspection uncertainty is presented in his work, where a Bayesian updating scheme is used. Wang and Majid [13] have performed a reliability analysis for an offshore oil platform plant and modeled its major preventive maintenance activities using the delay-time technique. They have minimized the system downtime using an optimal periodic inspection policy.

Currently there is a well-established literature on delaytime models where inspections may or may not be perfect. During the past four decades, a large number of papers on maintenance optimization models have been published, many of them written by highly skilled mathematicians, but without paying enough attention to the practicality aspects $[1,14]$. This paper uses the simple classical delaytime model and tries to develop a model applicable for the real-word maintenance problems, after implementing certain necessary modifications and extensions. However, there are a variety of maintenance optimization works for different structures and systems which have used other concepts. There is another work that has used a concept similar to the delaytime concept: Castanier and Rausand [14] have claimed to try to narrow the gap between the theory and practice of maintenance optimization by using the PF interval model, essentially proposed for the Reliability Centered Maintenance (RCM), to optimize the preventive replacement of a subsea oil pipeline. Their model can be used to determine the inspection interval that minimizes the average maintenance cost per unit time. Although they have mentioned a linear degradation trend for the internal coating of pipeline, the assumption of perfect repair has finally allowed for using the results of renewal theory. In other words, the structure (pipeline) lifetime is approximately taken as infinite and optimal periodic (uniform) inspection schedule is calculated. In the work by Dawotola et al. [15] a risk-based maintenance optimization of a petroleum pipeline is achieved by minimizing the total economic loss of failure while taking the human risk and maintenance budget as constraints. Their proposed framework is utilized for maintenance planning of a very long cross-country petroleum pipeline system and the optimal inspection interval for periodic planning is determined.

Speaking of suitable maintenance policies for the structures, it is clear that PM activities are eminently suitable since such structures usually have important societal and economical functions and their failures can generally be considered as catastrophic. More than this, for any PM program, inspection is a basic and vital activity because it provides information on the status of system under maintenance. The information can then be used for making the proper decision regarding the repair, replacement, or other maintenance activities.

Recently, Wang [16] has provided an overview of the recent advances in delay-time-based maintenance modeling and highlighted future research directions.

In this paper we have used the nonhomogenous Poisson process (NHPP) for modeling the deterioration process. This extension to the delay-time model was first done by Christer and Wang [8]. Again, they have minimized the total expected cost per unit time. The proposed model in this paper, unlike the most delay-time-based models found, focuses on finding the optimal aperiodic inspection schedule while the lifetime of the structural system is limited. The NHPP formulation suitably captures the deterioration process so that the model can also be considered as an extension to the applications of delay-time models. Also, because the lifetime is fixed and limited in the model, the renewal theory approximation is not used and unlike the other works, the downtime of the system is minimized via finding an optimal aperiodic inspection schedule.

The structure of the remaining parts of this paper is as follows. In Section 2 we introduce the notation and terminology and the assumptions of the model. The detailed formulation of the delay-time-based model is presented in Section 3. In Section 4 numerical study is conducted to show the behavior and results of the proposed model for a typical inspection planning problem. Further discussions and also the potential areas of work are brought in Section 5 .

\section{Assumptions, Notations, and Parameters}

In this section we emphasize the important details of the problem to be solved and list the assumptions and notations of the model for reference. 
Consider a system with the specified lifetime $L$. We want to find the optimal times of inspections that minimize the system downtime during its lifetime. The number of inspection activities is limited, implementing a budget constraint for the optimization problem. Thus the problem is to plan for a prespecified number of inspections $m$ to be performed so that while deterioration is present the system undergoes minimum total downtime. Below we have mentioned the most important assumptions of the delay-time-based model.

(i) The system under study is considered as single unit.

(ii) Inspections take place at times $t_{1}, t_{2}, \ldots, t_{m}$. System is new at $t_{0}=0$ and the end of its life will be at $t_{m+1}=L$. Thus the decision vector of the optimization problem will be of the form $\mathbf{t}=\left(t_{1}, t_{2}, \ldots, t_{m}\right)$.

(iii) Only one failure (and defect) mode is analyzed for the system. A failure happens after a defect is initiated and the delay-time is passed.

(iv) Defects arising are independent of each other.

(v) Defects arise as a nonhomogeneous Poisson process and the rate of occurrence/arrival of defects (ROCOD) at time $t$ will be $\lambda(t)$.

(vi) The defects delay-time probability density function is $f(t)$ and its cumulative density function is $F(t)$. The delay-time distribution is independent of the time origin.

(vii) The mean time for performing an inspection is $d_{s}$. During inspection the system is in the down state.

(viii) The mean time for rectifying each defect found from inspections is $d_{r}$.

(ix) The mean time for repairing each failure that happened is $d_{f}$.

(x) There is only one type of inspection activity considered to be performed for the system. Thus the quality and level of inspections are similar.

(xi) Inspections are perfect and any defect present will be recognized.

(xii) Repairs are considered minimal. Thus the system returns to the state just before the failure happened.

Assuming parametric forms for $\lambda(t)$ and $f(t)$ we will establish our delay-time-based model for the system in the next section. Hence the optimal inspection schedule can be derived after solving the optimization problem.

\section{Model Formulation}

In the previous section all the necessary variables and parameters were defined. Now we should establish the objective function of the model. It is worth mentioning that since the defects arise as a nonhomogeneous Poisson process, the expected number of defects and failures arising over nonoverlapping intervals of equal length will generally be unequal. In other words, the only renewal point in the stochastic process is the system replacement time, that is, structure lifetime $L$.
The objective function which is the system total downtime ratio is defined as the ratio of the sum of downtime periods occurring for the system to the sum of downtimes plus operating times. It can be simply formulated as shown in

$$
D(t ; L)=\frac{T D(\mathbf{t} ; L)}{T I(\mathbf{t} ; L)} .
$$

In (1), the nominator $T D(\mathbf{t} ; L)$ and denominator $T I(\mathbf{t} ; L)$ are the system total downtime and total interval durations (operating plus downtime), respectively. Equations (2) and (3) are formulated to calculate these terms:

$T D(\mathbf{t} ; L)$

$$
\begin{aligned}
= & \sum_{i=1}^{m}\left(d_{f} \cdot E\left[N_{f}\left(t_{i-1}, t_{i}\right)\right]+d_{s}+d_{r} \cdot E\left[N_{s}\left(t_{i}\right)\right]\right) \\
& +d_{f} \cdot E\left[N_{f}\left(t_{m}, t_{m+1}\right)\right],
\end{aligned}
$$

$T I(\mathbf{t} ; L)$

$$
=\sum_{i=1}^{m}\left(\left(t_{i}-t_{i-1}\right)+d_{s}+d_{r} \cdot E\left[N_{s}\left(t_{i}\right)\right]\right)+\left(t_{m}, t_{m+1}\right) .
$$

There are three different terms in the summation used in (2) accounting for the downtime periods due to the unexpected failures, downtime period due to conduct of inspection, and downtimes needed for rectifying defects found at inspection intervals.

Similarly, the summation used in (3) accounts for the operating time (including downtimes related to repairs) of each interval, downtime period due to conduct of inspection, and downtimes needed for rectifying defects found at inspection intervals. We should remark that there are $m$ planned inspections at times $t_{1}, t_{2}, \ldots, t_{m}$ but there are $m+1$ intervals during the system lifetime: $\left(t_{0}, t_{1}\right),\left(t_{1}, t_{2}\right), \ldots,\left(t_{m-1}, t_{m}\right),\left(t_{m}, t_{m+1}\right)$. The point is that no inspection happens after the last interval so that the corresponding downtime is only due to the failures occurring. For this reason, the summations in (2) and (3) cannot be used for all of the intervals. Thus, the downtime and total interval time of the last interval must be calculated separately.

In order to evaluate the objective function, we have to calculate two expected value terms appearing in (2) and (3), $E\left[N_{f}\left(t_{i-1}, t_{i}\right)\right]$ and $E\left[N_{s}\left(t_{i}\right)\right]$, which are the expected number of failures occurring in the interval $\left(t_{i-1}, t_{i}\right)$ and the expected number of defects found at the inspection time $t_{i}$, respectively. From the property of the underlying Poisson process for defect arrivals it follows directly that the expected number of defects arising in $\left(t_{i-1}, t_{i}\right)$ is given by

$$
E\left[N_{d}\left(t_{i-1}, t_{i}\right)\right]=\int_{t_{i-1}}^{t_{i}} \lambda(u) d u .
$$

The failures which occur during each interval can be modeled as another nonhomogeneous Poisson process [11, 17]. The corresponding rate of occurrence/arrival of failures (ROCOF) can be obtained by

$$
\rho(t)=\int_{t_{i-1}}^{t_{i}} \lambda(u) f(t-u) d u \quad\left(t_{i-1} \leq t<t_{i}\right) .
$$


Because generally every defect does not lead to a failure, we can predict that the values of ROCOF function $\rho(t)$ are to be less than ROCOD function $\lambda(t)$. This can be also understood from the mathematical formulation of $\rho(t)$ in which the existence of the probability term $f(t-u)$ lessens $\lambda(t)$ in the integration process. Similarly, the expected number of failures occurring during interval $\left(t_{i-1}, t_{i}\right)$ is given by

$$
E\left[N_{f}\left(t_{i-1}, t_{i}\right)\right]=\int_{t_{i-1}}^{t_{i}} \rho(u) d u .
$$

It is now possible to find the expected number of identifiable defects at the inspection time $t_{i}$ using (7). Attention must be paid that in each interval defects which start to grow and become a failure will finish in two different ways: either causing a failure or being rectified at the moment of (perfect) inspection. Thus we will have

$$
E\left[N_{f}\left(t_{i-1}, t_{i}\right)\right]+E\left[N_{s}\left(t_{i}\right)\right]=E\left[N_{d}\left(t_{i-1}, t_{i}\right)\right] .
$$

Substituting the terms from the previous equations we will have

$$
\begin{aligned}
E\left[N_{s}\left(t_{i}\right)\right]= & \int_{t_{i-1}}^{t_{i}} \lambda(u) d u \\
& -\int_{t_{i-1}}^{t_{i}} \int_{t_{i-1}}^{t_{i}} \lambda(u) f(v-u) d u d v .
\end{aligned}
$$

Thus, using (6) and (8) the objective function given in (1) can be evaluated. The constraints for decision variables can be simply constructed with the below set of equations:

$$
\begin{gathered}
t_{1}>0 \\
t_{2}>t_{1}+d_{s} \\
t_{3}>t_{2}+d_{s} \\
\vdots \\
L=t_{m+1}>t_{m}+d_{s} .
\end{gathered}
$$

The obtained optimization problem is a single-objective constrained nonlinear problem with $m$ variables and $m$ linear constraints. Solving this type of problem analytically is normally very time-consuming since the variables appear in the integral lower and upper bounds. However, the numerical solution to the problem with a reasonable number of decision variables (below 100) can be obtained very quickly with ordinary computers. Because the main purpose of this paper is to develop mathematical maintenance models, instead of their solution algorithms, we will content ourselves with establishing the model and analyzing the solutions reported by computer software such as MATLAB. In the next section we develop various numerical examples and discuss different aspects of them in detail.

\section{Numerical Examples}

So far we have described the details of our stochastic delaytime-based model formulation and derived the required

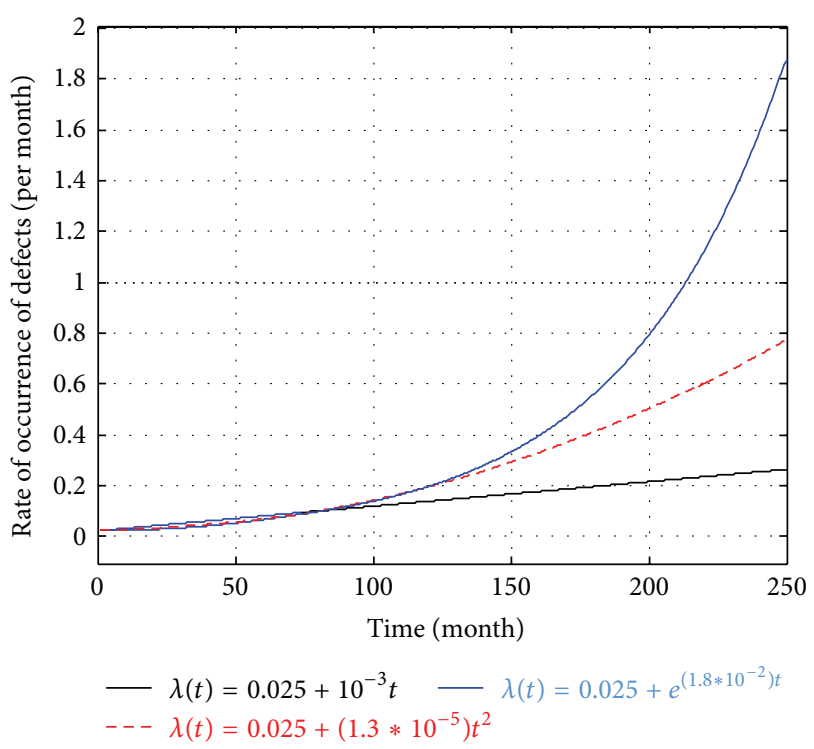

FIGURE 1: Linear, quadratic, and exponential functions used for the ROCOD.

TABLE 1: Values used for the model parameters.

\begin{tabular}{lcc}
\hline Parameter & Value & Unit \\
\hline$L$ & 240 & Month \\
$m$ & 30 & - \\
$d_{s}$ & 0.09 & Month \\
$d_{r}$ & 0.1 & Month \\
$d_{f}$ & 2 & Month \\
$\gamma$ & 0.0625 & Per month \\
\hline
\end{tabular}

expected values for computation of the objective function. In this section, we present different examples to show the performance of the model and the advantages of using an aperiodic inspection scheme for a deteriorating structure. We have assumed an exponential distribution $f(t)=\gamma \exp (-\gamma x)$ for the delay-time period as used traditionally in the previous works $[8,13,17]$. Three different function forms, linear, quadratic, and exponential, are considered for the rate of occurrence of defects $\lambda(t)$ which addresses different degradation trends. The initial value of ROCOD is equal for all of the three cases but the rates of growth vary significantly. Figure 1 shows the development of ROCOD during the structure lifetime according to these functions. All other parameters of the model are taken equal for the three cases so that the effect of severity of deterioration becomes clear. Values used for other parameters are typical for the group of systems under study, which are enlisted in Table 1.

Table 2 summarizes the results of using the model for the three described cases. The results are acquired using the MATLAB software. In addition to solving the optimization problem for the prescribed cases, the downtime ratio achieved using the traditional periodic inspection scheme is calculated and provided to enable us to make further comparisons. 
TABLE 2: Results summary for numerical examples.

\begin{tabular}{lccccc}
\hline $\begin{array}{l}\text { ROCOD } \\
\text { function } \\
\text { from }\end{array}$ & $\begin{array}{c}\text { Total downtime } \\
\text { ratio (\%) }\end{array}$ & $\begin{array}{c}\text { Powntime during } \\
\text { lifetime (month) }\end{array}$ & $\begin{array}{c}\text { Total downtime } \\
\text { ratio (\%) }\end{array}$ & $\begin{array}{c}\text { Optimized aperiodic schedule } \\
\text { Improvement in } \\
\text { downtime ratio (\%) }\end{array}$ & $\begin{array}{c}\text { Reduction in } \\
\text { downtime (month) }\end{array}$ \\
\hline Linear & 7.99 & 19.6 & 7.61 & 0.38 & 0.92 \\
Quadratic & 13.91 & 34.4 & 12.04 & 1.87 & 4.6 \\
Expon'l & 20.66 & 51.6 & 16.53 & 4.13 & 10.1 \\
\hline
\end{tabular}

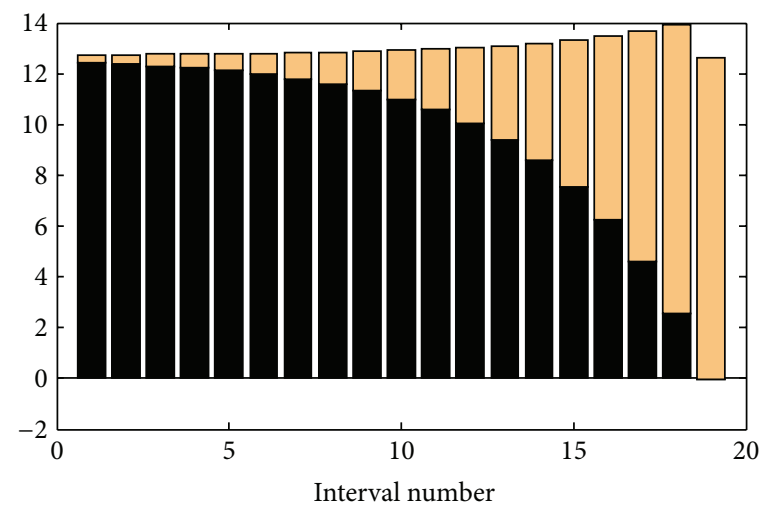

Interval operating time (month) Interval downtime (month)

(a)

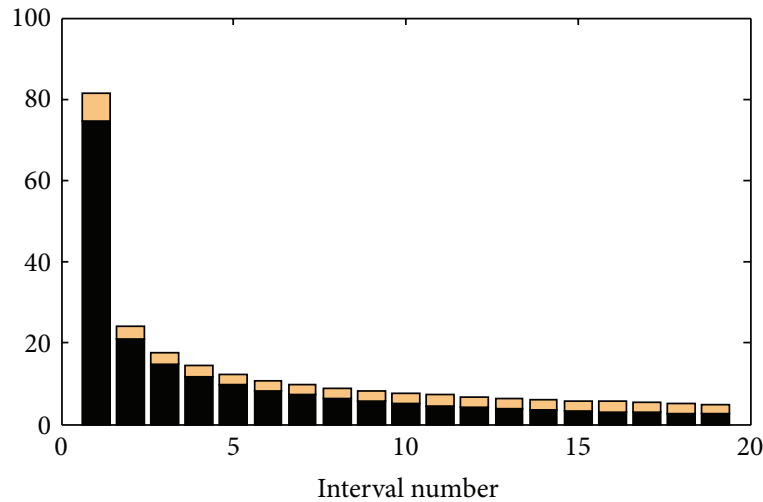

Interval operating time (month) Interval downtime (month)

(b)

FIGURE 2: Bar plot of downtimes and operating times for (a) traditional periodic inspection schedule and (b) optimized aperiodic schedule.

The important fact inferred from the results is that the more severe the damage caused by deterioration is, the more beneficial the implementation of an aperiodic inspection schedule will be. In detail, we initially observe that the total downtime ratio dramatically increases (from $7.99 \%$ to $20.66 \%$ ) as we use the exponential growth for the ROCOD, which would be indeed the more real choice for most mechanical and structural systems, instead of a linear growth function. However, the reduction in downtime duration after using the optimized aperiodic schedule is not significant (less than a month in 20 years) when the deterioration is not severe, that is, linear ROCOD. Quite the opposite, the effect of using the proposed aperiodic model is noteworthy for the exponential deterioration case, preventing more than 10 months of downtime, which is equivalent to 4.13\% improvement in the structure total downtime ratio.

Figure 2 gives more details about the evolution of inspection intervals as the structure ages, for the case of exponential ROCOD.

In Figure 2(a) it is seen that early inspection intervals feature very short downtime periods since the system defects and failures rarely happen; however, late maintenance intervals have long downtime durations due to the occurrence of failures and the time required for repairing them. Therefore the total sum of downtimes during the structure lifetime is rather large. This problem can be explained by the fact that the inspections done at the early stages are performed more frequently than required, thus revealing too few defects and avoiding negligible downtime caused by the consequent occurrence of failures. On the other hand, inspections at the final stages are done less frequently than needed; thus the downtime caused by corrective maintenance activities lessens the system operating time significantly. This problem is solved when we consider implementing the proposed aperiodic scheme for the structure. Figure 2(b) shows the results of an aperiodic optimized inspection schedule with exponential deterioration. It is seen that early intervals have very large operating time durations and inspections are done less frequently compared to the later inspections. Also, since those later inspections are done wisely and more frequently because of the unhealthy state of the structure, long downtimes are totally avoided. This, in fact, is achieved by lowering the number of defects and failures with reasonable and wise planning.

Expected number of defects found at inspections and expected number of failures occurring in each interval are shown in Figure 3. Again we can notice in Figure 3(a) that the expected number of failures and number of defects grow rapidly as the structure ages, mainly because the frequency of inspections is constant all the time neglecting the fact that final stages of structure lifetime are more critical and more frequent maintenance activities are required. But when we look at the numbers after using the aperiodic schedule in Figure 3(b) we can see that the expected numbers of both defects and failure are reduced to a large extent so that the operating times are increased noticeably. 


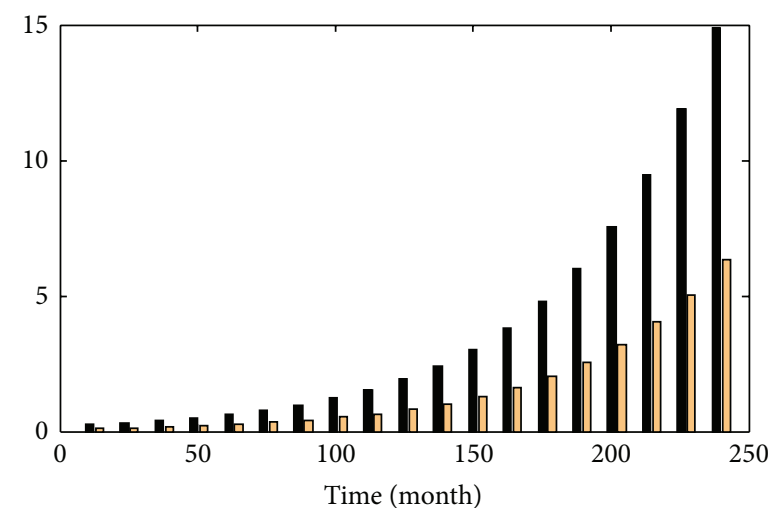

Expected number of defects Expected number of failures

(a)

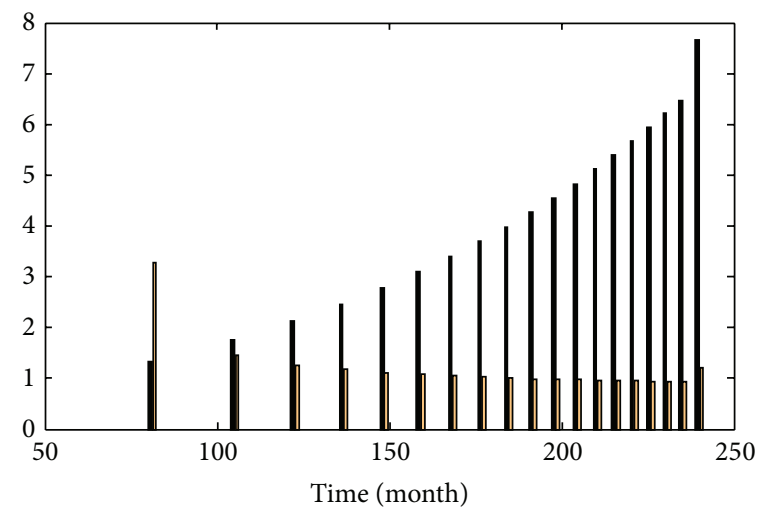

Expected number of defects Expected number of failures

(b)

FIGURE 3: Expected values of defects and failures related to each interval shown at inspection times for (a) traditional periodic inspection schedule and (b) optimized aperiodic schedule.

Another important matter is the value of $m$ which specifies the number of inspections to be conducted during the structure lifetime. So far we have considered it to be fixed and predetermined. However, we should not neglect the important role it plays. As we pointed out previously, this value basically implies a budget constraint for the structure; that is, since the inspection activities are inherently costly in many cases for infrastructures they must be planned and conducted based on budget limitations. On the contrary, their effect on avoiding system downtime is major especially if carried out in time. Figure 4 shows the improvement in system total downtime ratio for the above example with exponential deterioration when the number of inspections increases. The values are calculated both for the case of traditional periodic schedule and for the proposed optimized schedule. Initially we can notice the sharp decrease in the downtime ratio as we conduct more inspections. One underlying reason is the assumption of short downtime for conducting the inspections that adds to the defect-rectifying feature of inspections. We can also observe that for every value of $m$ the downtime ratio achieved by the aperiodic schedule is considerably lower than the periodic schedule, showing the advantage accrued by the proposed model. As the final point, although the trend is always declining, the rate of decline is not constant, being high for smaller values of $m$ and low for larger values. This is particularly important from a cost-benefit viewpoint since managers and decision makers can have a trade-off between the cost of extra inspections and the benefit brought by increased operating time.

\section{Conclusions}

In this paper we have proposed a new model to handle the problem of maintenance planning for structures undergoing deterioration. Using the nonhomogeneous Poisson process the deterioration phenomenon is implied in the model.

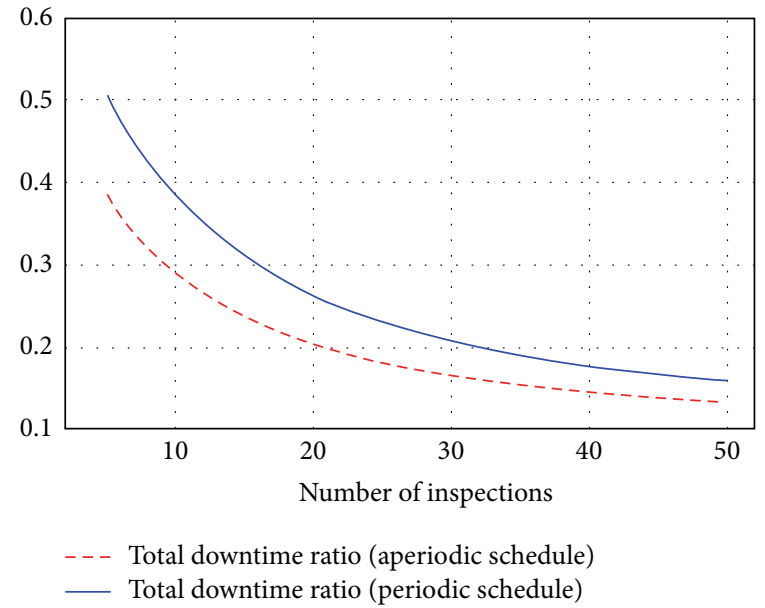

FIGURE 4: Reduction in downtime versus the number of inspections carried out during structure lifetime.

Whereas most previous works have used the classical renewal theory for modeling the occurrence of failures and other maintenance actions, minimal repair assumption in this model leads to the fact that the only renewal point in the stochastic process is the system replacement time. Also, the notion of delay-time concept is innovatively used for optimizing the inspection schedule; that is, modifications have been made both to the objective function and to the expected values to fit for the problem under study. The results of the numerical examples approve the superiority of the optimized aperiodic schedule.

Thorough analysis of the numerical studies reveals that the accrued benefit of the proposed model is substantially large when the resources are scarcer and the deterioration is more severe. In other words, when the costs of conducting inspections are high and the structure is in an unhealthy state, 
implementation of the proposed optimized schedule can save a lot of money and time from the cost-benefit and safety viewpoints.

Since the model is a basic general model for deteriorating structures and systems, suggested future work can be implementing more specific case-based modifications. For example, inspection uncertainties are present in many industrial applications and inclusion of imperfect inspections is very helpful. Also, modeling is done for a single unit system, but in many cases the system is multicomponent and reserved redundant units and their effect can be added to the model.

\section{Conflict of Interests}

The authors declare that there is no conflict of interests regarding the publication of this paper.

\section{References}

[1] D. M. Frangopol, M. J. Kallen, and J. M. van Noortwijk, "Probabilistic models for life-cycle performance of deteriorating structures: review and future directions," Progress in Structural Engineering and Materials, vol. 6, no. 4, pp. 197-212, 2004.

[2] T. Nakagawa, Maintenance Theory of Reliability, Springer, London, UK, 2005.

[3] S. Kumar, R. Dandotiya, R. Kumar, and U. Kumar, "Inspection frequency optimization model for degrading flowlines on an offshore platform," International Journal of Reliability, Quality and Safety Engineering, vol. 15, no. 2, pp. 167-180, 2008.

[4] A. Christer, "Innovative decision making," in Proceedings of the NATO Conference on the Role and Effectiveness of Theories of Decision in Practice, 1976.

[5] M. Abdel-Hameed, "Inspection and maintenance policies of devices subject to deterioration," Advances in Applied Probability, vol. 19, no. 4, pp. 917-931, 1987.

[6] M. Abdel-Hameed, "Correction to: 'inspection and maintenance policies of devices subject to deterioration," Advances in Applied Probability, vol. 27, no. 2, p. 584, 1995.

[7] P. Thoft-Christensen and J. D. Sorensen, "Optimal strategy for inspection and repair of structural systems," Civil Engineering Systems, vol. 4, no. 2, pp. 94-100, 1987.

[8] A. H. Christer and W. Wang, "A delay-time-based maintenance model of a multi-component system," IMA Journal of Management Mathematics, vol. 6, no. 2, pp. 205-222, 1995.

[9] M. D. Pandey, "Probabilistic models for condition assessment of oil and gas pipelines," NDT and E International, vol. 31, no. 5, pp. 349-358, 1998.

[10] S. Okumura, A. K. S. Jardine, and H. Yamashina, "An inspection policy for a deteriorating single-unit system characterized by a delay-time model," International Journal of Production Research, vol. 34, no. 9, pp. 2441-2460, 1996.

[11] S. Okumura, "An inspection policy for deteriorating processes using delay-time concept," International Transactions in Operational Research, vol. 4, no. 5-6, pp. 365-375, 1997.

[12] W. Wang and A. H. Christer, "Solution algorithms for a nonhomogeneous multi-component inspection model," Computers \& Operations Research, vol. 30, no. 1, pp. 19-34, 2003.

[13] W. Wang and H. B. A. Majid, "Reliability data analysis and modelling of offshore oil platform plant," Journal of Quality in Maintenance Engineering, vol. 6, no. 4, pp. 287-295, 2000.
[14] B. Castanier and M. Rausand, "Maintenance optimization for subsea oil pipelines," International Journal of Pressure Vessels and Piping, vol. 83, no. 4, pp. 236-243, 2006.

[15] A. W. Dawotola, T. B. Trafalis, Z. Mustaffa, P. H. A. J. M. van Gelder, and J. K. Vrijling, "Risk-based maintenance of a crosscountry petroleum pipeline system," Journal of Pipeline Systems Engineering and Practice, vol. 4, no. 3, pp. 141-148, 2013.

[16] W. Wang, "An overview of the recent advances in delay-timebased maintenance modelling," Reliability Engineering and System Safety, vol. 106, pp. 165-178, 2012.

[17] M. Ben-Daya, S. O. Duffuaa, A. Raouf, J. Knezevic, and D. AitKadi, Handbook of Maintenance Management and Engineering, Springer, London, UK, 2009. 

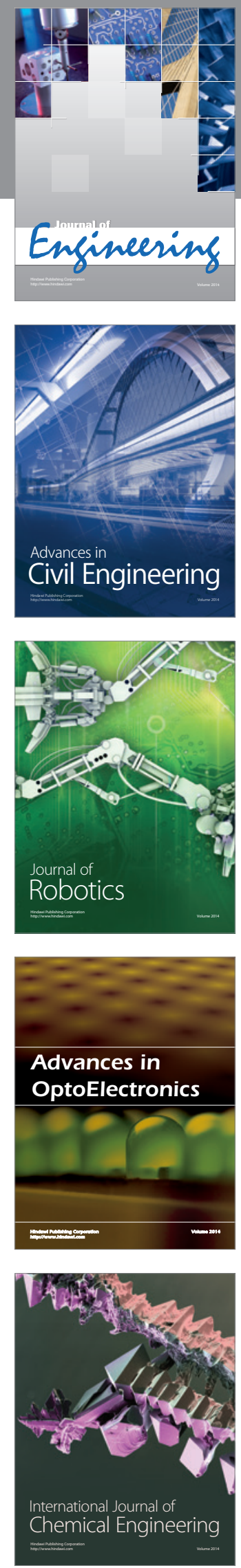

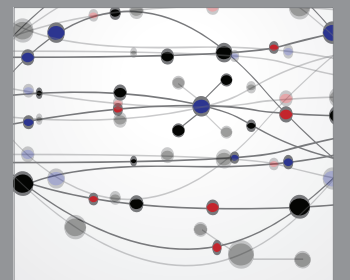

The Scientific World Journal
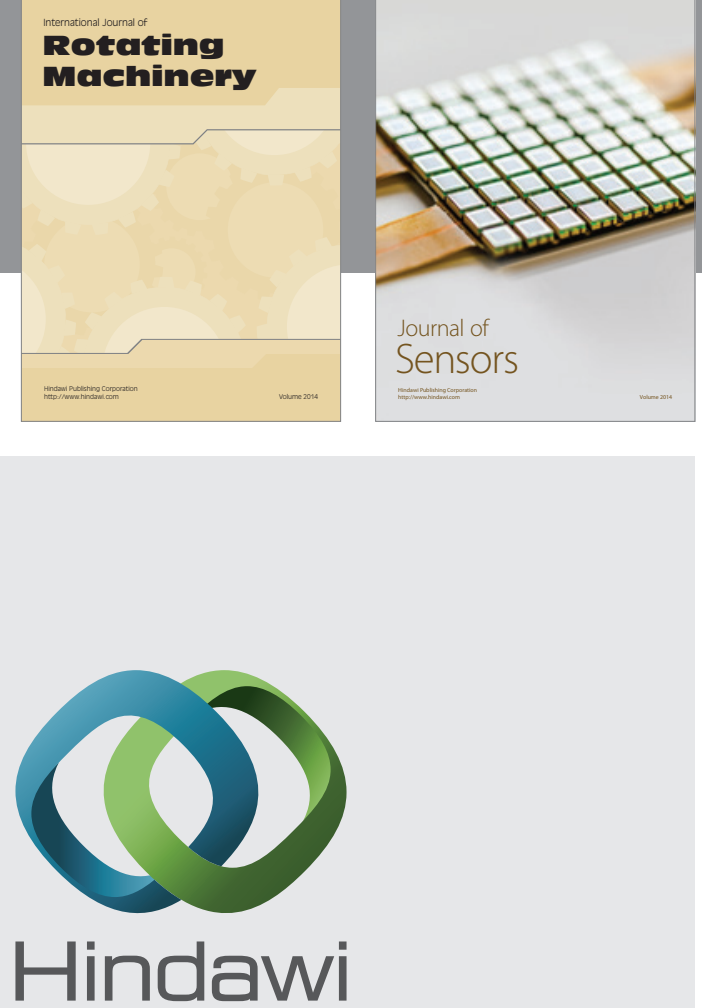

Submit your manuscripts at http://www.hindawi.com
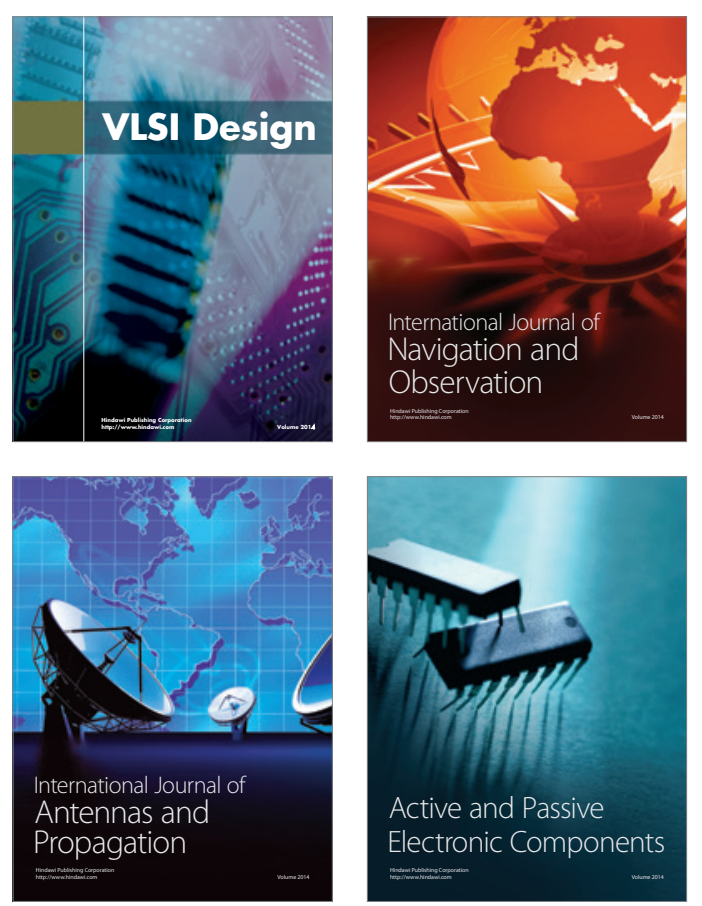
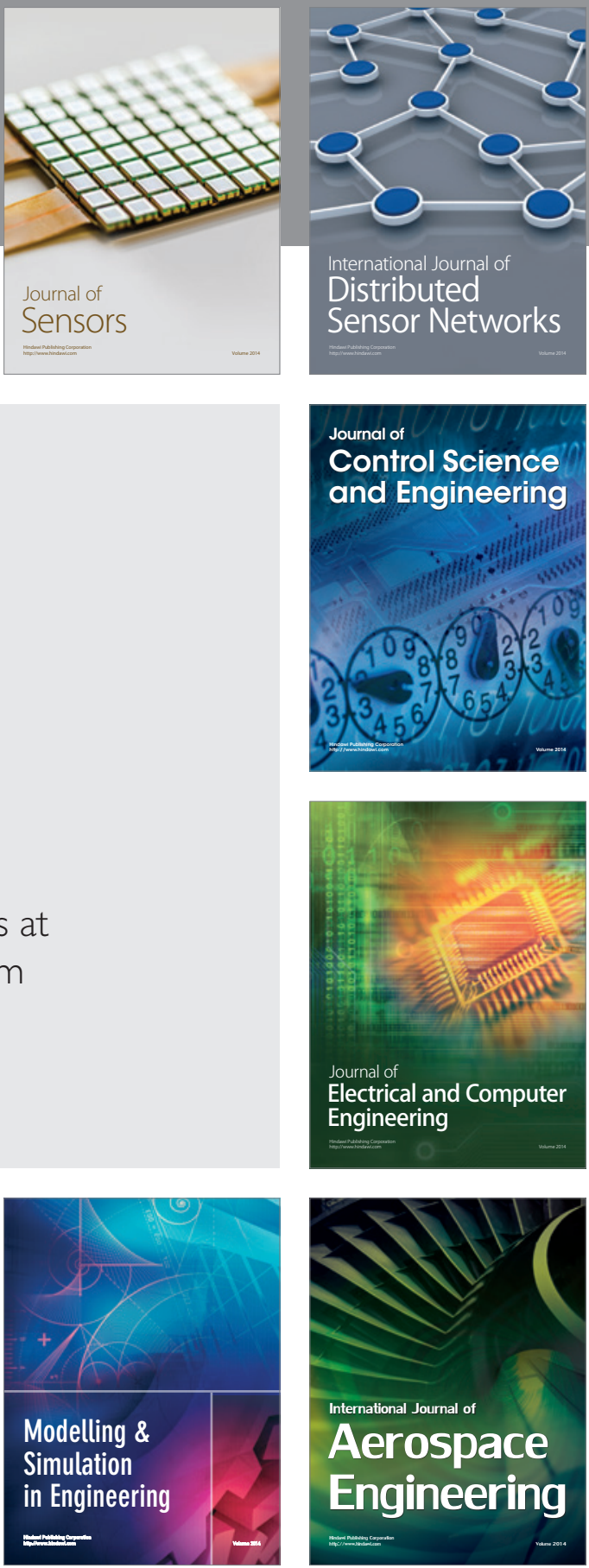

Journal of

Control Science

and Engineering
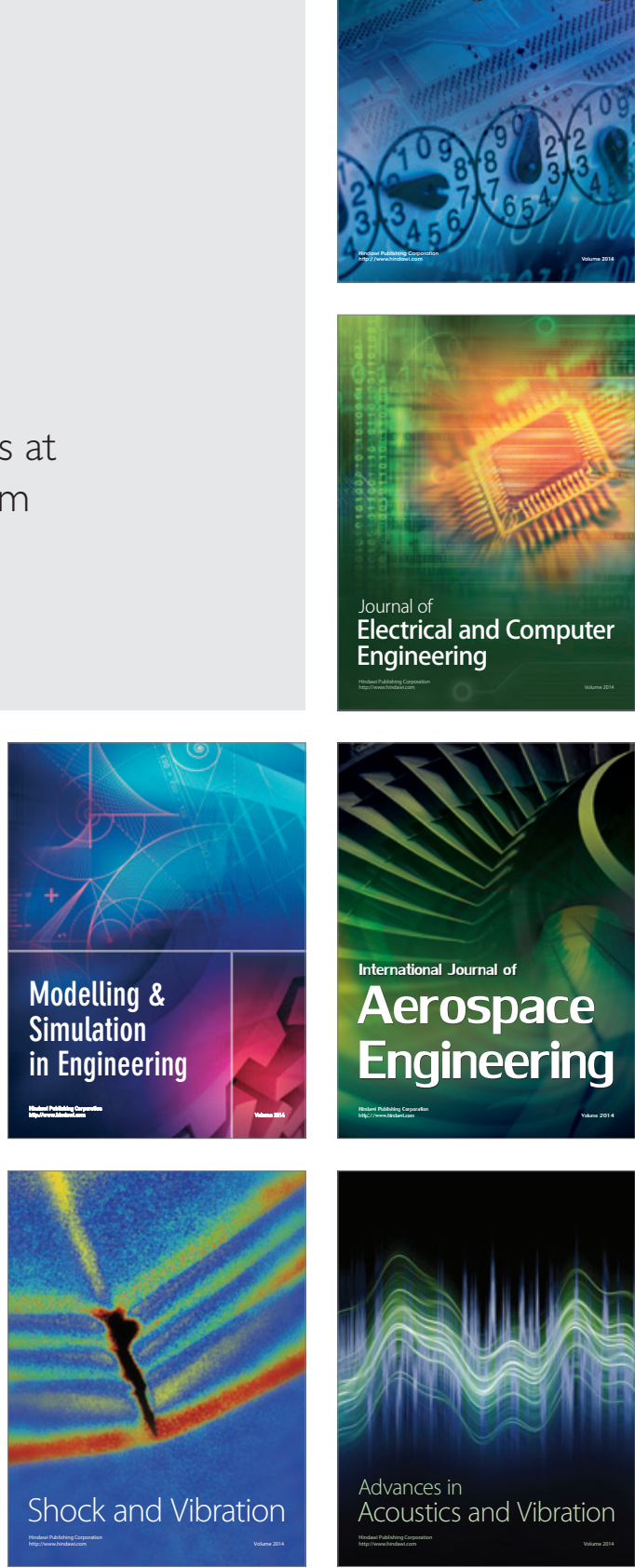